\title{
RETROCAVAL URETER - A CASE REPORT
}

\author{
CHOWDHURY MSA ${ }^{1}$, RAHMAN MM ${ }^{2}$, KARMAKER U ${ }^{3}$, BELAL MT $^{4}$, BHUIYUN MHK $^{5}$
}

\begin{abstract}
:
Retrocaval ureter is a rare congenital urologic anomaly. It occurs due to the persistence of the right subcardinal veins during embryologic development. Its presence should be suspected with the finding of a characteristic $S$-shaped deformity on intravenous or retrograde pyelography. Today, a definitive diagnosis can be made noninvasively using multi-slice CT imaging or MRI. Intervention is indicated in the presence of functionally significant obstruction leading to pain or renal function deterioration.
\end{abstract}

Key words: Retrocaval ureter, loin pain.

J Dhaka Med Coll. 2014; 23(2) : 259-261.

\section{Introduction:}

Retrocaval ureter may lead to extrinsic obstruction of the ureter. Although commonly referred to as circumcaval or retrocaval ureter, a more appropriate term may be preureteral vena cava, because it is due to a congenital abnormality in development of the vena cava. The term retrocaval is now primarily used to describe ureters that simply course behind the IVC and reemerge laterally.

Formation of the infrahepatic vena cava is based upon the development and regression of three pairs of embryonic veins: the posterior cardinal, the supracardinal, and the subcardinal. It is postulated that the normally developed IVC results from persistence of the right subcardinal vein suprarenally and the right supracardinal infrarenally. The posterior cardinal veins persist as the common iliac veins. The anastomosis between the right subcardinal and supracardinal vein crosses anterior to the fetal ureter. In normal development, this connection regresses, and the supracardinal vein persists as the infrarenal IVC. The prevailing theory of the development of this anomaly is that the subcardinal vein persists as the infrarenal IVC, thus crossing anterior to the midportion of the ureter and resulting in its circumcaval course $^{1}$.
The reported incidence of circumcaval ureter is approximately 1 in 1100 . There is a 2.8 -fold male predominance. Symptomatic patients typically present in the third or fourth decade of life ${ }^{2}$. This vascular anomaly is not always associated with ureteral obstruction ${ }^{3}$. The majority involve the right ureter, although leftsided circumcaval ureter has been reported in association with a duplicated IVC and in association with situs inversus ${ }^{4}$. Other genitourinary anomalies may be present. Circumcaval right ureter with a retrocaval horseshoe isthmus has been reported ${ }^{5}$.

\section{Case report:}

A 7 years old male child got admitted into the department of Urology in Dhaka Medical College Hospital with the complaints of pain in the right flank and occasional burning micturition with fever since birth. According to statement of the patient's mothe $r$ the presenting complaints started since birth with pain in the right flank, which is mild and dull in nature with no radiation, started gradually and last for few hours, aggravated by taking fluid and relieved with taking rest and voiding, not associated with fever or vomiting. Occasionally this child has been suffering from burning micturition and fever that last for few days and relieved by taking some medications; there was total 4-5 episodes since birth.

1. Dr. Md. Shafiqul Alam Chowdhury, Associate Professor of Urology, DMCH, Dhaka.

2. Dr. Md. Mostafizur Rahman,Assistant Professor of Urology,DMCH, Dhaka.

3. Dr. Uttam Karmaker, Assistant Professor of Urology, DMCH, Dhaka.

4. Dr. Md. Towhid Belal, Medical Officer of Urology, DMCH, Dhaka.

5. Dr. Md. Humayun Kabir Bhuiyun, Medical Officer of Urology, DMCH, Dhaka.

Correspondence: Dr. Md. Shafiqul Alam Chowdhury, Associate Prof. of Urology, DMCH, Dhaka. 


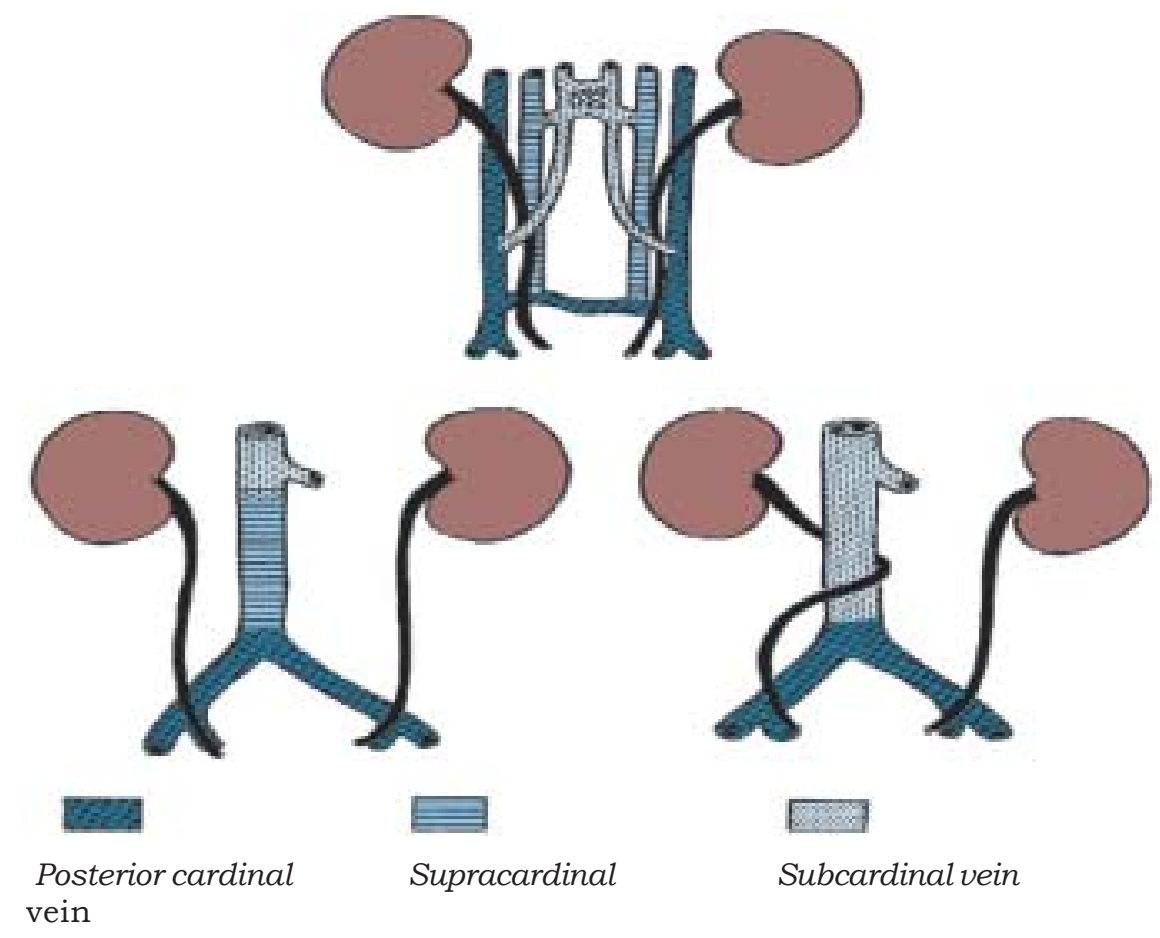

Figure 1. Relationship between the development of the infrarenal portion of the inferior vena cava and the retrocaval ureter.

He has no history of hematuria, calcuria, pyuria, low grade fever, night sweats, weight loss, cough, hemoptysis, chest pain or bony pain. He is normotensive, non-diabetic, nonasthmatic and not taking any regular medication. There is no history of any surgical procedure. He came from a low socio-economic group, lives in a rural area with his parent and one sister and all of them are apparently healthy. He has no known allergy to any diet or drug and he is fully immunized as per EPI schedule.

On general physical assessment the child is good looking, mentally sound and cooperative. Body built and nutrition is average. He is not anemic, non-icteric; temperature is $97^{\circ} \mathrm{F}$, pulse $110 \mathrm{bpm}$ and regular; all others parameters are normal. On genitourinary system examination- renal angles are non-tender, kidneys are not palpable, suprapubic region is not full, non-tender; hernial orifices are intact; external genitalia, scrotum and perinium are normal. Others systemic examination reveals no abnormality. Urine culture shows no growth, USG shows mild to moderately hydronephrotic right kidney and excretory urography shows type I retrocaval ureter.
After optimization, counselling and consent patient underwent retgrogade pyelography followed by open surgical pyeloureterostomy

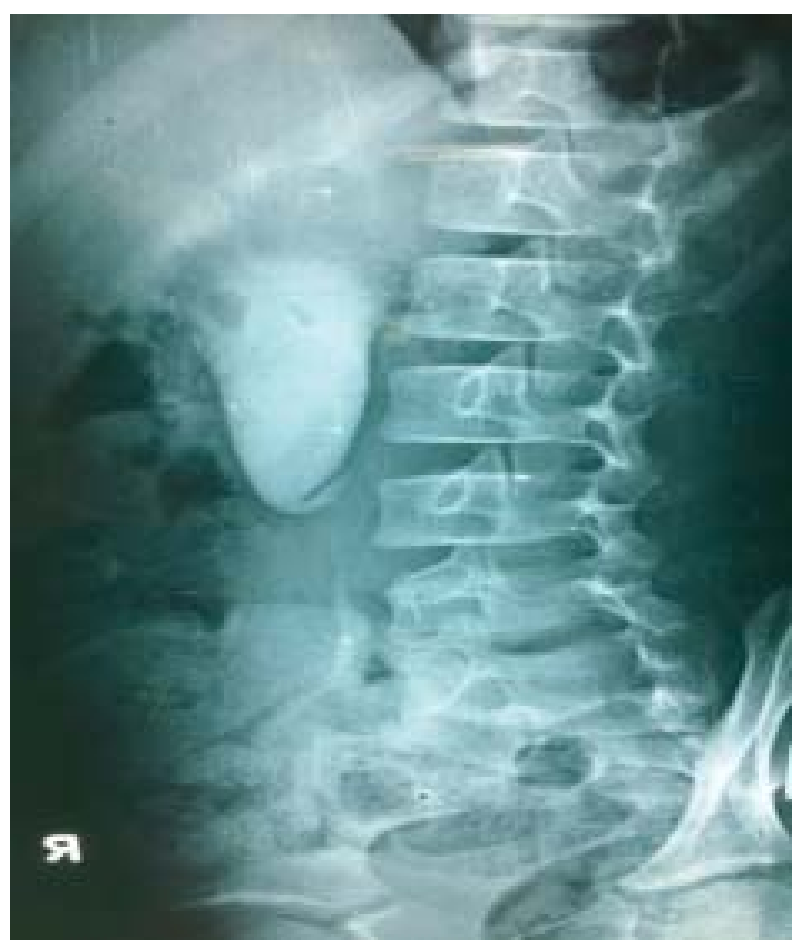

Figure 2. Type 1 retrocaval ureter on IVU. 


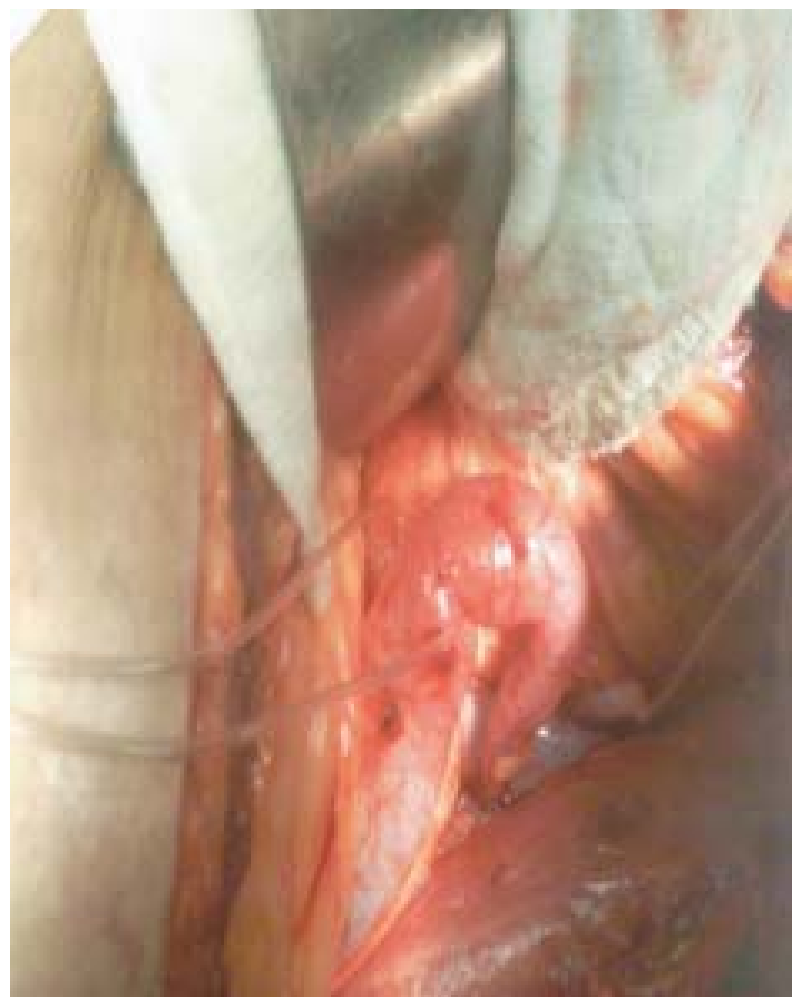

Fig.-3: Retrocaval ureter, peroperative view.

through flank approach with a JJ stent keeping in situ. Post operative recovery was excellent and JJ stent was removed after 4 weeks. First follow up after one month of JJ stent removal shows no pain, healthy surgical scar mark with no organism on culture of urine.

\section{Discussion:}

Retrocaval ureter is classified using different parameters based on intravenous urography or retrograde pyelographic findings. It is classified as a ureter with an S-shaped, fish hook, or shepherd's crook appearance as type I. The ureter typically overlies or is medial to the lower lumbar vertebral processes and eventually crosses anterior to the iliac vessels, where it then assumes a normal distal course in this setting. A less-angulated "sickle-shaped" ureteral deformity is classified as type II. The type I is associated with a greater degree of obstruction and hydronephrosis than type II. Another classification scheme is based on the level of obstruction; type I crosses at the level of the third lumbar vertebra, and type II crosses at the level of the ureteropelvic junction. The latter may be confused with ureteropelvic junction obstruction. However, an intrinsic ureteral abnormality may be contributory as histologic studies of involved ureteral segments have demonstrated fibrotic changes.

Patient may be asymptomatic and discovered on imaging studies performed to evaluate patients with nonurologic problems; or abdominal/flank pain, recurrent urinary tract infection and hypertension may be the initial symptoms and signs. The diagnosis can be confirmed with CT and MRI. Diuretic renography is used to confirm the presence and determine the functional impact of obstruction.

Treatment is indicated when there is presence of obstruction, pain or complications. The ureter is divided proximally and at the distal point, from which it emerges lateral to the IVC. A spatulated ureteroureterostomy is performed. This has been performed by open, laparoscopic, and retroperitoneoscopic approaches and is best dictated by the surgeon's experience ${ }^{6}$. Recently, pure robotic repair of retrocaval ureter has also been described successfully ${ }^{7}$.

\section{Conclusion:}

Retrocaval ureter results from the persistence of right subcardinal veins. The standard repair of retrocaval ureter is open surgical pyeloureterostomy or ureteroureterostomy. Retrocaval ureter can be diagnosed using intravenous or retrograde pyelography or 3-D CT. Procedural intervention is indicated in the presence of functionally significant obstruction, and both open and laparoscopic approaches can be successfully applied.

\section{Referrences:}

1. Singh, I. and Strandhoy, JW. Pathophysiology of Urinary Tract Obstruction. In: Wein, AJ. Editor. Cambell-Walsh Urology. $10^{\text {Th }}$ ed. Pennsylvania; 2012. P. 1118-20.

2. Zhang, XD., Hou, SK., Zhu, JH., Wang, XF., Meng, GD. and Qu, XK. Diagnosis and Treatment of Retrocaval Ureter. Eur Urol, 1990; 18: 207-210.

3. Crosse, JEW., Soderdahl, DW., Teplich, SK., et al. Diagnosis and Treatment of the Circumcaval Ureter. J Urol, 1975; 113: 743-6.

4. Watanabe, M., Kawamura, S., Nakada, T., et al. Retrocaval Ureter: Two Case Reports. J Urol, 1991; 145: 1047-48.

5. Knutson, T. and Hawas, B. Horseshoe Kidney with a Circumcaval Ureter. Scand J Urol Nephrol, 2004; 38: 348-50.

6. Bagheri, F., Pusztai, C., Szanto, A., et al. Robotic Reconstruction of Retrocaval Ureter. J Urol, 2009; 74: 1242-5.

7. Hemal, AK., Nayyar, R., Gupta, NP., et al. Robotic Reconstruction of Retrocaval Ureter. J. eururo, 2008; 54: 1443-6. 